\title{
1 Higher-order olfactory neurons in the lateral horn supports odor valence and 2 odor identity coding in Drosophila
}

4 Sudeshna Das Chakraborty, Hetan Chang, Bill S. Hansson" and Silke Sachse ${ }^{\# *}$ 5

6 Department of Evolutionary Neuroethology, Max Planck Institute for Chemical Ecology, Hans-

7 Knoell-Str. 8, 07745 Jena, Germany

8

9 "These authors share senior authorship

10

11 *For correspondence: ssachse@ice.mpg.de

12

13

14

15

16 


\section{Abstract}

18

19

20

21

22

23

24

25

26

27

28

29

30

31

32

33

34

35

36

37

38

39

41

42

43

Understanding neuronal representations of odor-evoked activities and their progressive transformation from the sensory level to higher brain centers features one of the major aims in olfactory neuroscience. Here, we investigated how odor information is transformed and represented in higher-order neurons of the lateral horn, one of the higher olfactory centers implicated in determining innate behavior, using Drosophila melanogaster. We focused on a subset of third-order glutamatergic lateral horn neurons (LHNs) and characterized their odor coding properties in relation to their presynaptic partner neurons, the projection neurons (PNs) by two-photon functional imaging. We found that odors evoke reproducible, stereotypic and odorspecific response patterns in LHNs. Notably, odor-evoked responses in these neurons are valencespecific in a way that their response amplitude is positively correlated with innate odor preferences. We postulate that this valence-specific activity is the result of integrating inputs from multiple olfactory channels through second-order neurons. GRASP and micro-lesioning experiments provide evidence that glutamatergic LHNs obtain their major excitatory input from uniglomerular PNs, while they receive an odor-specific inhibition through inhibitory multiglomerular PNs. In summary, our study indicates that odor representations in glutamatergic LHNs encode hedonic valence and odor identity and primarily retain the odor coding properties of second-order neurons.

\section{Introduction}

Insects are the most successful taxon among the whole animal kingdom in terms of their distribution and ability to survive in a multitude of environmental conditions. Largely they rely on their olfactory sense to carry out their fundamental goal directed behaviors, such as food navigation, mating, ovipositing or escape from predators. The powerful ability to detect odor cues, to evaluate the information efficiently with a relatively small number of neurons and to transform

the neuronal signal into an appropriate behavioral output, makes the insect olfactory system a 2 premier model system for olfactory research. Numerous studies have investigated the neuronal 43 representation of odors at successive neuronal layers from the periphery to higher brain levels 4 using Drosophila melanogaster as a model organism (Bhandawat et al., 2007; Ng et al., 2002; Root et al., 2007; Schmuker et al., 2007; Schmuker \& Schneider, 2007; Seki et al., 2017; Wilson 
et al., 2004). Although much progress has been made in understanding odor coding at the antennal lobe (AL) level (Bhandawat et al., 2007; Galizia, 2014; Ng et al., 2002; Wilson et al., 2004), the coding strategies and processing mechanisms of higher brain centers still remain largely elusive. In this regard, the lateral horn (LH) has recently gained attention as a crucial signal processing center integrating both innate as well as learned behavioral information (Das Chakraborty \& Sachse, 2021). Several studies during recent years have advanced our understanding of the anatomical and functional properties of higher-order lateral horn neurons (LHNs) regarding odor processing (Das Chakraborty \& Sachse, 2021; Dolan et al., 2019; Frechter et al., 2019; Jeanne et al., 2018; Varela et al., 2019). The generation of several LH cell type specific lines, characterization of polarity and neurotransmitter identity of LHNs as well as the establishment of detailed EM connectomic data sets have led to a significant progress in the field to study the function of specific LHN classes (Dolan et al., 2019; Frechter et al., 2019). The LH is comprised of three categories of neurons, which include LH input neurons (LHINs, which are mainly olfactory projection neurons (PNs) along with mechanosensory, thermosensory and gustatory neurons), LH local neurons (LHLNs) and LH output neurons (LHONs) (Bates et al., 2020; Dolan et al., 2019; Frechter et al., 2019). In terms of PN-LHN connectivity, the olfactory PNs deriving from individual glomeruli of the AL form stereotyped and conserved connections with certain LHNs (Fişek \& Wilson, 2014; Jeanne et al., 2018; Jefferis et al., 2007; Marin et al., 2002; Wong et al., 2002). Although all kind of connections are possible, PNs having similar odor tuning patterns are prone to target similar LHN types (Jeanne et al., 2018). Certain pairs of narrowly tuned glomeruli encoding ecologically relevant odors and eliciting specific kinds of behavior, such as courtship, aggregation or food seeking, converge onto the same LHN types and have been shown to be over-represented in the LH in terms of synaptic densities (Bates et al., 2020; Jeanne et al., 2018). Furthermore, a high amount of divergence has also been described to occur at the level of PN to LHN connectivity (Huoviala et al., 2018). Altogether these complex connectivity patterns in addition to direct pooling of feed-forward inputs from PNs innervating different glomeruli results in broader tuning patterns of LHNs compared to their presynaptic PNs (Bates et al., 2020; Frechter et al., 2019). In addition to the observed broadly tuned LHNs, narrowly tuned LHNs also exist, which receive input from a single type of $\mathrm{PN}$ and which are assumed to be further modulated by odorant selective inhibition through inhibitory neurons (Fişek \& Wilson, 2014). Although several studies agree that odors are compartmentalized in the LH based on either their chemical 
77 identity (Frechter et al., 2019), behavioral significance (Bates et al., 2020; Jeanne et al., 2018;

78 Jefferis et al., 2007) or hedonic valence (Strutz et al., 2014; Tanaka et al., 2004), it still remains

79 controversial how the odor information is transformed from the PN to the LHN level and which

80 odor features are coded by subtypes of LHNs.

81

82

83

84

85

86

87

88

89

90

91

92

93

94

95

96

97

98

99

100

101

102

103

104

105

In the present study, we aimed to elucidate the odor coding and processing strategies of LHNs by investigating how a neuronal subset of particular neurotransmitter identity encodes different odor features in the LH and how this representation is correlated to their presynaptic partner neurons in the AL, the uni- and multiglomerular PNs (uPN, mPN). Using photoactivatable GFP, we first identified diverse clusters of LHNs based on their different neurotransmitter identities and further focused our detailed analysis exclusively on glutamatergic LHNs. Using in vivo two-photon functional imaging, we characterized several aspects of odor-evoked activity in these neurons, such as odor-specific response patterns, reproducibility of repeated stimulations as well as stereotypy across different individuals. We could successfully demonstrate that attractive and aversive odors are clearly segregated and that their response amplitudes are positively correlated with the innate behavioral preference to an odor. We also dissected how excitatory input from uPNs and odor-specific inhibition from mPNs contribute to the fine-tuning of odor-specific response patterns of LHNs. Altogether this study demonstrates a significant role of glutamatergic LHNs regarding olfactory processing and extends our knowledge about the transformation processes of neuronal information taking place from the periphery to higher brain levels, such as the LH. (1)

\section{Results}

\section{Identification of higher-order neurons in the $\mathbf{L H}$}

To unravel distinct neuronal circuits in the LH, we expressed photoactivatable GFP (UAS-C3PA

GFP) (Ruta et al., 2010) under different Gal4 driver lines with various neurotransmitter and neuromodulator identities (i.e. Cha-Gal4, GAD1-Gal4, dVGlut-Gal4, TH-Gal4 and tdc2-Gal4)

(Figure 1A, B). To precisely define the upper and lower limit of the LH, we labeled uPNs using GH146-QF, QUAS-td tomato in the background as a landmark in every experiment. Using twophoton excitation we photoactivated C3PA-GFP throughout the LH neuropil with laser pulses of 
106

107

108

109

110

111

112

113

114

115

116

117

118

119

120

121

122

123

124

125

126

127

128

129

130

131

132

133

134

135

$760 \mathrm{~nm}$ in the in vivo fly brain. Photoactivation of the LH using driver lines for cholinergic (ChaGal4) and GABAergic neurons (GAD1-Gal4) revealed clusters of LHNs with labeled somata along with cholinergic and GABAergic PNs, respectively. The labeled cholinergic and GABAergic LHN clusters were located ventrolaterally to the LH. Selective labeling of glutamatergic neurons in the LH by photoactivation using the $d V G l u t-G a l 4$ driver line revealed several clusters of LHNs cell bodies positioned dorsomedial, ventrolateral and ventral to the LH with major neurite tracts entering the $\mathrm{LH}$ at distinctive locations. Glutamatergic LHNs are comprised of a large number of labeled somata $(\sim 40)$ and a few ventrolateral and ventral clusters possessing long neuronal tracts (Figure 1B). The labelled glutamatergic LHNs may include both LHLNs and LHONs (Frechter et al., 2019). Photoactivation of the LH using drivers for dopaminergic (TH-Gal4) and octopaminergic neurons (tdc2-Gal4) revealed the presence of both kinds of LHNs (i.e. LHLNs, LHONs) in the LH (Figure 1A). These neurons innervate the LH sparsely with a very long neuronal tract and their somata positioned far away from the LH. Since we observed a high abundance of glutamatergic neurons in the LH, which were non-overlapping with second-order neurons (i.e. PNs) deriving from the AL, we confined our further experiments on this subset of LHNs to elucidate their functional properties regarding odor coding.

\section{Glutamatergic LHNs reveal reproducible, stereotypic and odor-specific responses that} emerge from the PN level

To elucidate how odors are represented in the LH, we monitored odor-evoked functional responses of glutamatergic LHNs. To do so, we expressed the genetically encoded calcium sensor UASGCaMP6f (Chen et al., 2013) that has a high sensitivity and low basal fluorescence under the control of $d V G l u t$-Gal4. In order to accurately and consistently identify a comparable focal plane for functional imaging across different animals, we expressed QUAS-td tomato in uPNs using GH146-QF in the background as a landmark. We performed in vivo two-photon functional imaging from the glutamatergic LHNs at three different focal planes to comprehensively monitor odor responses throughout the LH (i.e. upper, middle and lower plane). We used a panel of 14 ecologically relevant odors at a concentration of $10^{-3}$, which have been shown to be behaviorally either attractive or aversive (Knaden et al., 2012). Our imaging data show that each odor evoked a specific activity pattern across all three focal planes in the LH (Figure 2A). Since the LH appears 
136

137

138

139

140

141

142

143

144

145

146

147

148

149

150

151

152

153

154

155

156

157

158

159

160

161

162

163

164

rather homogenous and lacks clear morphological landmarks, we used a grid approach for imaging data analysis (Figure 2B) (see Methods for details) and determined the $\Delta \mathrm{F} / \mathrm{F}$ to analyze the neuronal activity across odors for each animal. At first, we compared the reproducibility of the evoked responses of these glutamatergic LHNs to two sequential presentations of each odor. This analysis of neuronal activity for repeated odor stimulations clearly showed that the odor-evoked responses were highly reproducible both qualitatively and quantitatively across trials (Figure 2C), which is further depicted by a high correlation coefficient across repeated stimulations for all odors (Figure 2D). Hence, although the odor responses appear generally broad, each odor induced an unique response pattern in these glutamatergic LHNs (Figure 2D). Furthermore, a cluster analysis based on the linkage distance between the spatial patterns of each odor response reveals that the majority of the replicates for each odor are clustered together, confirming that individual odors are represented in an odor-specific manner by glutamatergic LHNs (Figure 2F). To investigate whether the observed odor response properties of glutamatergic LHNs derived from their presynaptic partner neurons, we determined the odor representations of uPNs in the LH to the same odor set. Previous studies based on the innervation pattern of PNs reported a compartmentalization in the LH either based on the hedonic valence of an odor, i.e. attractive versus aversive odors (Seki et al., 2017), the odor category, i.e. pheromones versus food odors (Jefferis et al., 2007) or with regard to the behavioral 'odor scene' (Bates et al., 2020; Jeanne et al., 2018). However, whether an odor-specific response map exists at the uPN level in the LH has so far not been unambiguously shown with a functional approach. We therefore expressed UAS-GCaMP6f under control of GH146-Gal4 and monitored odor-evoked responses at the two-photon microscope from the same three focal planes as used for the LHN imaging experiments. Notably, when we compared the odor response properties of uPNs with those of LHNs we observed that the PNs possess a significantly reduced correlation coefficient as compared to LHNs, implying that odors are better segregated at the uPN level in the LH than at the third-order neuron level (Figure 2E). In addition, a cluster analysis based on the linkage distance between the spatial patterns of each odor response at the PN level confirms that uPNs also reveal odor-specific response patterns in the LH (Figure 2G). Altogether, these observations clearly demonstrate and signify that uPNs as well as glutamatergic LHNs exhibit an odor-specific response map in the LH. 


\section{Response amplitude of glutamatergic LHNs reflects odor valence}

167

168

169

170

171

172

173

174

175

176

177

178

179

180

181

182

183

184

185

186

187

188

189

190

191

192

193

194

195

196

Next, we investigated whether hedonic valence is still preserved in the LH level in this particular subset of LHNs as it has been described for both types of PNs, i.e. uPNs and mPNs (Knaden et al., 2012; Strutz et al., 2014). We therefore quantified and pooled the correlation coefficients of all odor responses of glutamatergic LHNs to all attractive and aversive odors and compared those that shared the same valence (i.e. within valence) as well as for odors having an opposing valence (i.e. across valence) for all three focal planes measured (Figure 3A). Interestingly, we observed that the responses to odors with the same valence were significantly more similar, depicted by a higher correlation coefficient, compared to the odor responses across valence. In order to visualize the odor representations in a multidimensional space, we employed a principal component analysis (PCA) of the odor response profiles by taking into account two parameters, the spatial response patterns as well as the intensity of odor-evoked responses by each odor. As expected, the attractive and aversive odor responses were segregated significantly throughout the $\mathrm{LH}(* \mathrm{p}<0.05$, one way ANOSIM) confirming that odors are indeed categorized according to their behavioral value by these third-order glutamatergic LHNs (Figure 3B). Notably, when only the response amplitude was considered, it was obvious that attractive odors in general evoked a stronger activity in glutamatergic LHNs compared to responses to behaviorally aversive odors across all three focal planes (Figure 3C and D). Next, we examined whether odor responses of second-order uPNs also reflect the hedonic valence of an odor within the $\mathrm{LH}$ as it has been shown for the AL level previously (Knaden et al., 2012). A PCA of the odor response profile of uPNs revealed that the attractive and aversive odors are clearly segregated $(* * p<0.005$, one way ANOSIM) at the PN level also in the LH (Figure 3E). In order to investigate if, similar to LHNs, the response magnitude in uPNs contributes to the observed clear segregation, we averaged the overall response activities to attractive and aversive odors across all three planes. Indeed, we observed a similar trend, meaning that uPNs responded stronger to attractive odors than to aversive ones in the LH (Figure 3D and F). This observation was novel and unexpected and tempted us to postulate that the flies' innate odor preference might be determined by the response amplitude of uPNs and glutamatergic LHNs. To address whether the odor-evoked response strength is correlated with behavioral preference, we performed T-maze assays to the same odor set as used for the functional imaging experiments. As shown in previous studies, flies displayed different levels of attraction and aversion to individual odors (Knaden et al., 2012; MacWilliam et al., 2018; Min et al., 2013; 
197

198

199

200

201

202

203

204

205

206

207

208

209

210

211

212

213

214

215

216

217

218

219

220

221

222

223

224

225

226

Strutz et al., 2014). Plotting the olfactory preference indices of all tested odors with the response strengths of glutamatergic LHNs indeed revealed a significant correlation (Figure 3G) showing that odors eliciting stronger LHN activity induce stronger attraction, while odors that elicit lower LHN responses induce a stronger aversion. Interestingly, the response strength of uPNs did not correlate significantly with the olfactory preference indices (Figure 3H), although uPNs activity displayed a valence-specific representation (Figure 3C). This observation suggests that the activity in the subset of glutamatergic LHNs may contribute significantly to the observed behavioral odor preferences. However, this correlation does not confirm any causality between the response strength of glutamatergic LHNs and the behavioral output. To test a causal relationship, it would be necessary to silence or artificially activate the glutamatergic LHNs and to determine the functional consequences with regard to behavioral valence. However, since the line $d V G l u t$ Gal4 is broadly expressed throughout the whole brain, genetic manipulations would not be confined to the subset of glutamatergic LHNs and would therefore be difficult to evaluate. One might argue that the observed differential LHN responses emerge already at the sensory neuron level and are not the result of neuronal processing within the AL or LH. To examine this idea, we obtained odor-evoked response of olfactory sensory neurons (OSNs) to individual odors from the DoOR datasbase (Münch \& Galizia, 2016) and plotted the response strengths of glutamatergic LHNs either against the overall OSN activity (Figure 3I) or the number of ORs (olfactory receptors) activated by a specific odor (Figure 3J). Interestingly, we could not observe any correlation in either case, indicating that the differential LHN activity observed here is not deriving from the OSN level, but is the result of second-order neuronal processing. Notably, the comparison between uPNs and LHNs responses also did not show any correlation (Figure 3K), further suggesting that the differential activity observed in the glutamatergic LHNs is not a mere relay of the AL input through uPNs, but rather processed by either mPNs or other LHNs to generate the observed graded odor responses in higher-order glutamatergic LHNs.

\section{Polarity and connectivity of glutamatergic LHNs with uPNs and mPNs}

To determine the neuronal polarity of the glutamatergic LHNs, we expressed pre- and postsynaptic markers (UAS-sypGC3, UAS-homerGC3) under the control of dVGlut-Gal4. Immunohistochemistry followed by confocal scanning demonstrated that these neurons possess 
227

228

229

230

231

232

233

234

235

236

237

238

239

240

241

242

243

244

245

246

247

248

249

250

251

252

253

254

255

256

both, presynaptic dendrites and postsynaptic axon terminals in the LH (Figure 4A, left and right panel), indicating that the cell cluster of glutamatergic LHNs probably comprises both LHLNs as well as LHONs (Dolan et al., 2019). The presence of presynaptic terminals suggests that these neurons provide input to other LHONs, LHLNs or even perform a feedback inhibition onto PNs. To assess the neuronal connectivity of these neurons with excitatory and inhibitory PNs at the morphological level in detail, we employed the GRASP technique (Feinberg et al., 2008). Initially, we employed two split-GFP fragments, tagged to the extracellular domain of the CD4 transmembrane protein (hereafter referred to as $C D 4-G R A S P$ ). To determine the connectivity between uPNs and glutamatergic LHNs, we expressed one fragment of GFP in glutamatergic LHNs, using $d$ VGlut-Gal4 and the other fragment in uPNs, using GH146-LexA (Figure 4B, left panel). Next, to examine whether mPNs and glutamatergic LHNs are synaptic partner neurons, we employed a similar approach by using $d V G l u t-L e x A$ and MZ699-Gal4 (Figure 4B, right panel). In both cases we observed a clear neuronal connectivity to the glutamatergic LHNs. However, since CD4-GRASP is not synaptically targeted and can potentially lead to false positive signals at nonsynaptic locations, we employed in addition a GFP 1-10 fragment, which is tagged to the synaptic protein neurexin, resulting in an enhancement of synaptic specificity (Fan et al., 2013; Shearin et al., 2018). Neurexin is present predominantly at the presynaptic site (Fan et al., 2013; Xing et al., 2018), but also occurs along with postsynaptic terminals (Taniguchi et al., 2007). When we performed GRASP by expressing neurexin in glutamatergic LHNs, we observed a clear GFP signal in the LH (Figure 4C, left panel) implying that glutamatergic LHNs provide presynaptic output onto uPNs and therefore convey a feedback inhibition to the AL. Such a feedback connection needs to be verified in future experiments. Next, in order to determine the synaptic connectivity between glutamatergic LHNs and mPNs, we expressed neurexin in mPNs and observed distinct GFP puncta in the LH implying that also mPNs form presynaptic and probably postsynaptic connections with glutamatergic LHNs (Figure 4C, right panel).

Glutamatergic LHNs receive major excitatory input from uPNs and an odor-specific inhibition from mPNs

In order to further elucidate the neural connectivity and to verify our GRASP experiments, we determined the functional connectivity of glutamatergic LHNs with uPNs and mPNs by transection 
experiments using two-photon laser-mediated micro-dissection. To manipulate the input from uPNs, we micro-lesioned the iACT tract entering the LH and compared the odor-evoked responses to vinegar and benzaldehyde from the glutamatergic LHNs before and after laser ablation (Figure 5, schematic upper panel). After transecting the iACT tract we observed that the odor-induced responses to both, vinegar and benzaldehyde were completely abolished across all three focal planes of the LH (Figure 5 A-A', B-B'") This finding implies that glutamatergic LHNs receive their major excitatory input from the AL through uPNs. To verify whether this complete elimination of the odor-evoked activity is not just a technical side effect of the laser ablation procedure, we also monitored odor responses from the glutamatergic LHNs in the other, untreated brain hemisphere before and after micro-lesioning, which proved not to be affected (Figure 5figure supplement 1). In order to examine the functional connectivity between mPNs and glutamatergic LHNs, we also transected the mACT tract entering the LH and monitored odor responses from glutamatergic LHNs before and after laser transection. Notably, transecting the mACT led to a significant increase in the odor-evoked responses in the middle and upper plane of the glutamatergic LHNs (Figure 5 C-C', D-D'). We further observed that the mACT transection affected the odor-evoked responses differentially in a way that the response increase was higher in the case of vinegar than for benzaldehyde. This observation indicates that the glutamatergic LHNs receive an odor selective inhibition from mPNs which is stronger for the food odor vinegar than for the repellent odor benzaldehyde.

\section{mPN- mediated inhibition onto glutamatergic LHNs contributes to odor specificity}

Next, we aimed to understand the impact of the observed odor-specific inhibition of mPNs with regard to the odor-specific responses of glutamatergic LHNs in the LH. To address this issue, we monitored odor-evoked responses in the glutamatergic LHNs to two repeated stimulations of vinegar and benzaldehyde (depicted as 1 and 2 in the schematic in Figure 6A, A'), followed by measuring LHN activity after micro-lesioning the mACT tract (depicted as 3 in Figure 6A, $\mathbf{A}^{\prime}$ ). Since, we observed a significant response increase following mPN-mediated inhibition only in the middle and upper plane (Figure 5C', C', D' and D'), we confined our analysis to these two areas. In order to determine whether the laser ablation of mACT alters also the odor-evoked spatial pattern in addition to an increase in response amplitude, we compared the correlation coefficients 
between the repeated responses before laser ablation to those after the transection. Our results revealed that removal of the inhibitory input from mPNs leads to a reduced correlation coefficient of the odor response patterns evoked by vinegar in LHNs (Figure 6B, B'). Interestingly, such an effect was not observed in the case of benzaldehyde although we observed a weak mPN-mediated inhibition for this odor before (Figure 6B, B'). Since the odor-evoked spatial patterns were modified after silencing the mPN input into the LH, we next wondered whether this also affected the odor specificity of the LHN responses. We therefore compared the correlation coefficients 'across odors' (i.e. between vinegar and benzaldehyde) before and after microlesion (Figure 6C). Strikingly, we observed that the odor-evoked response patterns of vinegar and benzaldehyde became increasingly similar after removal of the mPN-mediated inhibition depicted as an increased correlation coefficient after laser ablation. This finding suggests that the inhibitory input from mPNs onto glutamatergic LHNs is crucial to generate a distinct and odor-specific response pattern for individual odors at the LH level.

\section{Discussion}

Our study functionally characterizes a subset of glutamatergic higher-order neurons in the LH regarding odor coding and processing. We demonstrate that glutamatergic LHNs respond in a reproducible, stereotypic and odor-specific manner and these response properties emerge at the level of presynaptic uPNs (Figure 6D). Notably, the differential activity levels of glutamatergic LHNs to attractive and aversive odors are positively correlated to the olfactory behavioral preference indicating that these neurons are mainly tuned to attractive odors. The response features do not arise from the OSN level, but rather derive from local processing within the LH by integrating inputs from multiple olfactory channels through uPNs, which also show valence specific odor representation. Furthermore, laser transection experiments demonstrate that these

311 higher-order neurons receive their major excitatory input from uPNs and an odor-specific 312 inhibitory input from mPNs. Lastly, our data show that the observed mPN-mediated inhibition 313 seems to be required for generating an odor-specific response map in the LH (Figure 6D). 


\section{Glutamatergic LHNs respond in a stereotypic and odor-specific manner}

317 A growing body of evidence suggests the existence of an anatomical and functional stereotypy in 318 early processing centers of the insect olfactory pathway (Hildebrand \& Shepherd, 1997;

319 Korsching, 2002). This stereotypy becomes obvious first at the sensory neuron (OSN) level, where

320 OSNs expressing a certain OR target and converge on a stereotypic glomerulus resulting in a

321 conserved spatial map in the AL between different individuals (Wang et al., 2003; Wilson et al.,

322 2004). This anatomical stereotypy was shown to be retained at the postsynaptic PN level (Jefferis

323 et al., 2007; Tanaka et al., 2004).

Several studies support the notion that an anatomical stereotypy might also be present at

325

326

327

328

329

330

331

332

333

334

335

336

337

338

339

340

341

342

343

344

the level of the LH, in particular shown for the PN to LHN connectivity (Bates et al., 2020; Fişek \& Wilson, 2014; Jeanne et al., 2018; Jefferis et al., 2007). Along this line, functional studies have demonstrated that LHNs respond in a reproducible and stereotyped manner to odors and this stereotypy is a general feature of the LH (Fişek \& Wilson, 2014; Frechter et al., 2019). However, how an ensemble of LHNs integrates inputs from several olfactory channels, i.e. the presynaptic excitatory and inhibitory PNs, and whether each odor induces a specific and stereotyped response pattern in the LH was not clearly addressed before. In our study we demonstrate that each odor is represented by an odor-specific activity pattern in the LH, while the examined glutamatergic LHNs display broader tuning patterns than their presynaptic partner neurons. Although it has been assumed previously that odor specificity may not be encoded in higher-order brain centers (Grabe \& Sachse, 2017; Strutz et al., 2014), our findings are in accordance with a recent study by Frechter et al. (2019) who demonstrate the existence of 33 different LH cell types exhibiting stereotypic odor response properties with increased tuning breadth than PNs. The observed broader tuning breadth of LHNs could be due to their property of integrating various inputs from multiple odor channels, e.g. one LHN receives on average excitatory input from 5.2- 6.2 glomeruli (Frechter et al., 2019; Jeanne et al., 2018). Here, we observed that uPNs are more efficient in encoding odor identity than the glutamatergic LHNs in the LH (Figure 6D), whereas LHNs reveal an improved categorization of odors either based on behavioral significance, 'odor scene' or chemical group (Bates et al., 2020; Frechter et al., 2019; Jeanne et al., 2018). However, the distinct odor-specific response map by glutamatergic LHNs observed in our study suggests that the dimensionality of 
odor features might not get reduced but still retains information about the odor specificity at the third-order processing stage.

\section{Coding of hedonic odor valence}

349 Our functional imaging recordings revealed that odor valence is encoded by glutamatergic LHNs 350 leading to different activation strengths and patterns for attractive and aversive odors in the LH.

351 This observation is well in line with previous studies that have revealed that odor-evoked responses in higher brain centers are generally categorized according to certain odor features as already mentioned above. For example, using functional imaging or patch-clamp recordings of secondorder olfactory neurons revealed the existence of distinct attractive and aversive odor response domains in the LH formed by uPNs and mPNs (Seki et al., 2017; Strutz et al., 2014). Such a categorization according to hedonic valence is also visible in our study, when the odor-evoked responses of glutamatergic LHNs were plotted in a PCA, taking into account the spatial response patterns as well as the intensity of activity. Although no prominent spatial domain of attractive or aversive odors was evident in our recordings, we observed that attractive odors evoked a generally stronger activity when compared to aversive odors in this subset of LHNs. We noted a similar trend in second-order uPNs. However, their response strength was neither correlated with the olfactory preference determined in behavior nor the odor response properties of LHNs. The observed significant correlation between the amount of odor-evoked activity in glutamatergic LHNs to the behavioral valence of an odor leads us to postulate that the activity strengths of higherorder olfactory neurons to odor stimulation might determine the behavioral response - an assumption that needs to be tested in future studies.

Our study suggests that glutamatergic LHNs use different strategies to extract different odor-specific activity map and, 2) encoding the valence of an odor by integrating information from

370 multiple olfactory channels. It is an ongoing debate regarding how neurons in the LH evaluate an 371 odor stimulus. It can be noted that irrespective of how an animal detects, encodes and categorizes 372 an odor at different olfactory processing levels, the behavioral output is in any case a binary choice, 373 reflected in either to approach (positive) or to leave (negative), to copulate (positive) or to reject 
374 (negative), to oviposit (positive) or to find another suitable oviposition site (negative) (Galizia,

375 2014). Hence, based on the context or ecological relevance, an odor can just be evaluated either as

376 'pleasant' or 'unpleasant', which is well reflected by the response properties of glutamatergic

377 LHNs regarding their valence-specificity and their correlation between response strength and

378 behavioral odor preference.

Notably, such a correlation between response intensity and behavioral preference has also been observed in previous studies, where the amplitude of food odor-evoked activity in neuropeptide $\mathrm{F}$ (dNPF) neurons was found to strongly correlate with food odor attractiveness (Beshel \& Zhong, 2013). Another study that combined functional imaging with tracking of innate behavioral responses revealed that the behavioral output could be accurately predicted by a model summing up the normalized glomerular responses, in which each glomerulus contributes a small but specific part to the resulting odor preference (Badel et al., 2016). At the level of the LH, LHNs then integrate the olfactory information from the glomerular responses conveyed via uPNs and mPNs. In one of our previous studies we demonstrated that mPNs respond differently to attractive and aversive odors, and mediate behavioral attraction (Strutz et al., 2014). In this context our study complements this previous finding by showing that also uPNs display distinct valence-specific responses in the LH to attractive and aversive odors. Information from these two PN pathways become integrated and processed in the LH resulting in valence-specific activities in glutamatergic LHNs, which may in turn determine the relative behavioral preference.

mPN-mediated inhibition generates odor-specific response patterns in the LH

We demonstrate that mPNs inhibit the glutamatergic LHNs in an odor-selective manner leading to an odor-specific response pattern. According to our observations, glutamatergic LHNs receive a stronger inhibition from mPNs in response to the odor vinegar than to benzaldehyde. In the absence of this inhibition, we noted that in addition to an increased response amplitude and altered odor representation, the activity patterns of different odors became more strongly correlated and hence more similar. We therefore conclude that the $\mathrm{mPN}$-mediated selective inhibition on this 401 glutamatergic subset of LHNs is necessary to maintain odor specificity. Along this line, a previous 402 study has reported that mPNs provide an odor-selective input to vlpr neurons, another class of 
403

404

405

406

407

408

409

410

411

412

413

414

415

416

417

418

419

420

421

422

423

424

425

426

427

428

429

430

third-order LHNs (Liang et al., 2013). According to these authors, this odor-specific modulation depends on the nature of the odor and results from the stereotyped connectivity of mPNs in the AL as well as in the LH. Although this study provides evidence that uPNs are not presynaptically inhibited by mPNs, another study established that mPNs indeed inhibit uPNs in the LH, facilitating odor discrimination (Parnas et al., 2013). In addition to uPNs and vlpr neurons, our study identifies another class of recipient neurons (glutamatergic LHNs) that receives mPN-mediated odorantselective inhibition. The glutamatergic LHNs population in our study comprises glutamatergic LHONs as well as LHLNs (Dolan et al., 2019). Although we were not able to clearly differentiate the functional properties between different populations of LHONs and LHLNs, since we used a general marker for glutamatergic neurons, our study provides the first understanding of how odors are integrated, transformed and finally represented in the LH by an ensemble of glutamatergic LHNs.

Intriguingly, the neurotransmitter identity of this class of LHNs opens up another interesting aspect: Knowing that glutamate can act as an excitatory (Aungst et al., 2003; Das et al., 2011a) or inhibitory neurotransmitter (Liu \& Wilson, 2013), as well as a coincident detector (Das et al., 2011b), depending upon the receptors present in the postsynaptic neurons, further experiments are needed to reveal the consequences of glutamatergic LHN input onto their postsynaptic partner neurons. Certainly, the presence of an impressive amount of vesicular glutamate in the LH points towards a significant role of glutamatergic LHNs with regard to odor coding and processing at this higher brain center.

\section{Acknowledgments}

This research was supported through funding by the Max Planck Society and Alexander von Humboldt Foundation (grant to S.D.C). Stocks were obtained from the Bloomington Drosophila Stock Center and used in this study (NIH P40OD018537). We express our gratitude to Silke Trautheim for her excellent support in fly rearing. 


\section{Author contributions}

432

433

434

435

436

437

438

439

440

441

442

443

444

445

446

447

448

449

450

451

452

453

454

455

456

S.D.C and S.S designed research; S.D.C and H.C performed research; S.D.C, H.C and S.S analyzed data; B.S.H. provided intellectual and financial support, S.D.C, B.S.H. and S.S. wrote the paper.

\section{Declaration of Interests}

The authors declare no conflict of interest.

\section{Materials and methods:}

\section{Fly stocks}

Flies were raised on autoclaved cornmeal-yeast-sucrose-agar food in a 12-h light/dark cycle at $25^{\circ} \mathrm{C}$ incubator. The following lines have been used for functional imaging: $d$ VGlut-Gal4 (II) (Mahr \& Aberle, 2006) (Bloomington 26160), GH146-Gal4 (II) (Stocker et al., 1997) (from Leslie Vosshall's lab.), GH146-QF, QUAS mtd Tomato (BDSC 30037) and UAS-GCaMP6f (III) (Chen et al., 2013) (Bloomington 52869). For photoactivation experiments Cha-Gal4 (II) (Bloomington 6798), GAD1-Gal4 (II) (Ng et al., 2002) (Bloomington 51630), dVGlut-Gal4, TH-Gal4 (III) (Zhang et al., 2007) (from Mani Ramaswami’s lab), tdc2-Gal4 (II) (Koon et al., 2010) (NCBS, India) and UAS-C3PA (Ruta et al., 2010) (gift from Sandeep Datta) flies have been used. Wild type Canton-S flies have been used for behavioral experiments.

\section{Photoactivation}

UAS-C3PA was expressed under Cha-Gal4, GAD1-Gal4, dVGlut-GAL4, TH-Gal4 and tdc2-Gal4 in the background of GH146-QF, QUAS mtd Tomato for the photoactivation experiment. An initial pre-photoactivation scan of the whole LH area was taken at $925 \mathrm{~nm}$ with a $40 \times$ water immersion objective. The extent of the LH in z section was identified based on the innervation pattern of uPNs (visualized by GH146-QF, QUAS mtd Tomato). A region of interest in different focal planes covering the entire LH area was photoactivated for $\sim 1-2$ min followed by 2-min rest using $\sim 7 \mathrm{~mW}$ 
457

458

459

460

461

462

463

464

465

466

467

468

469

470

471

472

473

474

475

476

477

478

479

480

481

482

483

484

485

of $760 \mathrm{~nm}$ of laser. We allowed 10-15 min for photoactivated GFP to diffuse in more distal neural processes. The post-photoactivation scan was taken using the same set-up as used for the prephotoactivation scan.

\section{Functional imaging}

All functional imaging experiments were performed on 4-7 day old mated female flies. Flies were dissected for optical imaging according to the protocol by Strutz et al. (2014). Flies were briefly immobilized on ice and then mounted onto a custom-made Plexiglas stage with a copper plate (Athene Grids, Plano). A needle before the head was placed to stabilize the proboscis and to align the head properly. Protemp II composite (3M ESPE) was used to fix the head with the copper plate. We bent the anterior part of the fly's head with fine gold wire, and a small plastic plate having a round window was placed on top. We sealed the head with that plate using twocomponent silicone (Kwik Sil) and leaving the center part open to make a cut. The cuticle between the eyes and the ocelli was gently cut under saline $(130 \mathrm{mM} \mathrm{NaCl}, 5 \mathrm{mM} \mathrm{KCl}, 2 \mathrm{mM} \mathrm{MgCl}, 2$ $\mathrm{mM} \mathrm{CaCl}_{2}, 36 \mathrm{mM}$ saccharose, $5 \mathrm{mM}$ Hepes, $1 \mathrm{M} \mathrm{NaOH}, \mathrm{pH}$ 7.3). The cuticle was either bent forward and fixed to the silicon or removed. All fat, trachea, and air sacs were removed carefully.

Functional imaging was performed using a 2-photon laser scanning microscope (2PCLSM, Zeiss LSM 710 meta NLO) equipped with an infrared Chameleon UltraTM diode-pumped laser (Coherent, Santa Clara, CA, USA) and a 40× water immersion objective lens (W Plan-Apochromat 40×/1.0 DIC M27). The microscope and the laser were placed on a smart table UT2 (New Corporation, Irvine, CA, USA). The fluorophore of GCaMP6f was excited with $925 \mathrm{~nm}$. For each individual measurement, a series of 40 frames (corresponding to $10 \mathrm{~s}$ ) acquired at a resolution of $256 \times 256$ pixels was taken with a frequency of $4 \mathrm{~Hz}$. During the entire $10 \mathrm{~s}$ of recording each odor was delivered after $2 \mathrm{~s}$ (8 frames) for $2 \mathrm{~s}$ (8 frames). To cover the entire LH, images were acquired from three different focal planes (i.e. upper, middle and lower), each $15 \mu \mathrm{m}$ apart in $\mathrm{z}$ direction. GH146-QF, QUAS-td tomato was always used in the background to precisely locate the planes for all imaging experiments. A set of 14 odors was used including phenylacetaldehyde (PHA), vinegar (VIN), 2,3 butanedione (BUD), acetoin acetate (AAC), ethyl acetate (ETA), propionic acid (PAC) and gamma-butyrolactone (G-BUT) as attractive odors, 1-octanol (OCT), benzaldehyde (BEA), 
acetophenone (ACP), methyl salicylate (MSC), geosmin (GEO) and linalool (LIN) as aversive odors and cis-vaccenyl acetate (cVA) as a pheromone. All odors have been used at a concentration of $10^{-3}$ diluted in mineral oil. Vinegar was diluted in double distilled water. Flies were imaged for up to $1 \mathrm{hr}$, with a minimum inter-stimulus interval of one minute. For odor delivery we used a computer-controlled odor delivery system (described in (Mohamed et al., 2019)).

\section{Data and statistical analysis for functional imaging}

The LSM file obtained from the imaging software ZEN, was processed and analyzed in Fiji. 40 frames for each odor (40 frames X 14 odors) were opened and stitched together using 'Image $>$ Stacks $>$ Tools $>$ Concatenate' command. The file combining all the frames for all odors were movement corrected using 'Plug in>Registration>Stackreg>Rigid body' command. The entire LH area was encircled and cropped and divided into 65 pixels, each having $30 \times 30 \mu \mathrm{m}^{2}$ dimension. Using 'Analyze>Tools $>$ ROI manager $>$ more $>$ multi measure' command fluorescence intensity for every pixel for every frame for every odor was obtained. This huge data set was further processed using Excel software. Average $\Delta \mathrm{F} / \mathrm{F}$ was calculated for 10-18 frames for each pixel for each odor.

The $\Delta \mathrm{F} / \mathrm{F}$ for 65 pixels for 14 odors for one replicate was aligned in table format and processed using 'past' statistical software. The correlation coefficient for each odor pair using Euclidean distance was analyzed.

\section{2-Photon-mediated laser transection}

Transection of either the iACT or mACT tract of PNs was performed using GH146-QF, QUAS-td tomato as a guiding landmark. Transection of the PN tracts was done in one brain hemisphere of each fly only. The chosen area for transection was located using $925 \mathrm{~nm}$ of laser, close to the $\mathrm{LH}$ but far enough not to damage the neurites branching in the LH neuropil. $\sim 60 \mathrm{~mW}$ of $760 \mathrm{~nm}$ laser was provided at the chosen area at a pulse for 2-3 s for 2-3 times with $1 \mathrm{~s}$ time interval (Strutz et al., 2014). Successful transection was verified after visualization of a small bubble. As a control, the LH from the other, untreated brain hemisphere was imaged to stimulation with benzaldehyde 
514 before and after laser ablation to rule out that the laser transection of the specific targeted area

515 caused any non-specific damage to other brain areas.

\section{Behavioral assay}

518 T-maze experiments with 4-7 days old flies were carried out for the olfactory preference assay.

$519 \sim 30$ flies were starved for $24 \mathrm{hrs}$ in each vial containing wet filter paper. Mated males and females

520 were used for the behavioral assay. All odors were used at a concentration of $10^{-3}$, which is the

521 same as used for the functional imaging experiments. At each arm of the T-maze small filter papers

522 containing either $6 \mu \mathrm{l}$ of mineral oil (control) or an odor (odorant) were placed. Briefly cold 523 anaesthetized flies were released at the junction of horizontally placed T maze. After $\sim 30$ min flies

524 in each side of the arm were counted. The preference index was calculated as (odorant-control)/ 525 total number of flies (i.e., 30). Each odor has $\sim 20$ replicates.

\section{References:}

528

529

530

531

532

533

534

535

536

537

538

539

540
Aungst, J. L., Heyward, P. M., Puche, A. C., Karnup, S. V., Hayar, A., Szabo, G., \& Shipley, M. T. (2003). Centre-surround inhibition among olfactory bulb glomeruli. Nature. https://doi.org/10.1038/nature02185

Badel, L., Ohta, K., Tsuchimoto, Y., \& Kazama, H. (2016). Decoding of Context-Dependent Olfactory Behavior in Drosophila. Neuron, 91(1), 155-167. https://doi.org/10.1016/j.neuron.2016.05.022

Bates, A.S., Schlegel, P., Roberts,R.J.V., Drummond, N., Tamimi, I.F.M., Turnbull, R., Zhao, X., Marin, E.C., Popovici, P.D., Dhawan, S.,...., Jefferis.G.S. (2020). Complete Connectomic Reconstruction of Olfactory Projection Neurons in the Fly Brain. Current Biology : CB, 30(16), 3183-3199.e6. https://doi.org/10.1016/J.CUB.2020.06.042

Beshel, J., \& Zhong, Y. (2013). Graded encoding of food odor value in the Drosophila brain. Journal of Neuroscience, 33(40), 15693-15704. https://doi.org/10.1523/JNEUROSCI.260513.2013 
541

542

543

544

545

546

547

548

549

550

551

552

553

554

555

556

557

558

559

560

561

562

563

564

565

566

567

568

Bhandawat, V., Olsen, S. R., Gouwens, N. W., Schlief, M. L., \& Wilson, R. I. (2007). Sensory processing in the Drosophila antennal lobe increases reliability and separability of ensemble odor representations. Nature Neuroscience, 10(11), 1474-1482. https://doi.org/10.1038/nn1976

Chen, T.-W., Wardill, T. J., Sun, Y., Pulver, S. R., Renninger, S. L., Baohan, A., Schreiter, E. R., Kerr, R. a, Orger, M. B., Jayaraman, V., Looger, L. L., Svoboda, K., \& Kim, D. S. (2013). Ultrasensitive fluorescent proteins for imaging neuronal activity. Nature, 499(7458), 295300. https://doi.org/10.1038/nature12354

Das, A., Chiang, A., Davla, S., Priya, R., Reichert, H., VijayRaghavan, K., \& Rodrigues, V. (2011a). Identification and analysis of a glutamatergic local interneuron lineage in the adult Drosophila olfactory system. Neural Systems \& Circuits, 1(1), 4. https://doi.org/10.1186/2042-1001-1-4

Das Chakraborty, S., \& Sachse, S. (2021). Olfactory processing in the lateral horn of Drosophila. Cell and Tissue Research 2021 383:1, 383(1), 113-123. https://doi.org/10.1007/S00441020-03392-6

Das, S., Sadanandappa, M. K., Dervan, A., Larkin, A., Lee, J. A., Sudhakaran, I. P., Priya, R., Heidari, R., Holohan, E. E., Pimentel, A., Gandhi, A., Ito, K., Sanyal, S., Wang, J. W., Rodrigues, V., \& Ramaswami, M. (2011b). Plasticity of local GABAergic interneurons drives olfactory habituation. Proceedings of the National Academy of Sciences, 108(36), E646-E654. https://doi.org/10.1073/pnas.1106411108

Dolan, M.-J., Frechter, S., Bates, A. S., Dan, C., Huoviala, P., Roberts, R. J., Schlegel, P., Dhawan, S., Tabano, R., Dionne, H., Christoforou, C., Close, K., Sutcliffe, B., Giuliani, B., Li, F., Costa, M., Ihrke, G., Meissner, G. W., Bock, D. D., ... Jefferis, G. S. (2019). Neurogenetic dissection of the Drosophila lateral horn reveals major outputs, diverse behavioural functions, and interactions with the mushroom body. ELife, 8, 1-45. https://doi.org/10.7554/eLife.43079

Fan, P., Manoli, D. S., Ahmed, O. M., Chen, Y., Agarwal, N., Kwong, S., Cai, A. G., Neitz, J., Renslo, A., Baker, B. S., \& Shah, N. M. (2013). Genetic and neural mechanisms that inhibit 
570

571

572

573

574

575

576

577

578

579

580

581

582

583

584

585

586

587

588

589

590

591

592

593

594

595

Feinberg, E. H., VanHoven, M. K., Bendesky, A., Wang, G., Fetter, R. D., Shen, K., \& Bargmann, C. I. (2008). GFP Reconstitution Across Synaptic Partners (GRASP) defines cell contacts and synapses in living nervous systems. Neuron, 57(3), 353-363. https://doi.org/10.1016/J.NEURON.2007.11.030

Fişek, M., \& Wilson, R. I. (2014). Stereotyped connectivity and computations in higher-order olfactory neurons. Nature Neuroscience, 17(2), 280-288. https://doi.org/10.1038/nn.3613

Frechter, S., Bates, A. S., Tootoonian, S., Dolan, M. J., Manton, J., Jamasb, A. R., Kohl, J., Bock, D., \& Jefferis, G. (2019). Functional and anatomical specificity in a higher olfactory centre. ELife, 8, 1-39. https://doi.org/10.7554/eLife.44590

Galizia, C. G. (2014). Olfactory coding in the insect brain: Data and conjectures. European Journal of Neuroscience, 39(11), 1784-1795. https://doi.org/10.1111/ejn.12558

Grabe, V., \& Sachse, S. (2017). Fundamental principles of the olfactory code. BioSystems, 164, 94-101. https://doi.org/10.1016/j.biosystems.2017.10.010

Hildebrand, J. G., \& Shepherd, G. M. (1997). Mechanisms of olfactory discrimination: Converging evidence for common principles across phyla. Annual Review of Neuroscience, 20(1), 595-631. https://doi.org/10.1146/annurev.neuro.20.1.595

Huoviala, P., Dolan, M., Love, F., Frechter, S., Roberts, R., Mitrevica, Z., Schlegel, P., Bates, A. S., Aso, Y., Rodrigues, T., Cornwall, H., Stensmyr, M., Bock, D., Rubin, G., Costa, M., \& Jefferis, G. (2018). Neural circuit basis of aversive odour processing in Drosophila from sensory input to descending output. BioRxiv. https://doi.org/10.1101/394403

Jeanne, J. M., Fişek, M., \& Wilson, R. I. (2018). The Organization of Projections from Olfactory Glomeruli onto Higher-Order Neurons. Neuron, 98(6), 1198-1213.e6. https://doi.org/10.1016/j.neuron.2018.05.011

Jefferis, G. S. X. E., Potter, C. J., Chan, A. M., Marin, E. C., Rohlfing, T., Maurer, C. R., \& Luo, L. (2007). Comprehensive Maps of Drosophila Higher Olfactory Centers: Spatially Segregated Fruit and Pheromone Representation. Cell, 128(6), 1187-1203. 
https://doi.org/10.1016/j.cell.2007.01.040

597

598

599

600

601

602

603

604

605

606

607

608

609

610

611

612

613

614

615

616

617

618

619

620

621

622

Knaden, M., Strutz, A., Ahsan, J., Sachse, S., \& Hansson, B. S. (2012). Spatial Representation of Odorant Valence in an Insect Brain. Cell Reports, 1(4), 392-399. https://doi.org/10.1016/j.celrep.2012.03.002

Koon, A. C., Ashley, J., Barria, R., DasGupta, S., Brain, R., Waddell, S., Alkema, M. J., \& Budnik, V. (2010). Autoregulatory and paracrine control of synaptic and behavioral plasticity by octopaminergic signaling. Nature Neuroscience 2010 14:2, 14(2), 190-199. https://doi.org/10.1038/nn.2716

Korsching, S. (2002). Olfactory maps and odor images. In Current Opinion in Neurobiology. https://doi.org/10.1016/S0959-4388(02)00348-3

Liang, L., Li, Y., Potter, C. J., Yizhar, O., Deisseroth, K., Tsien, R. W., \& Luo, L. (2013). GABAergic Projection Neurons Route Selective Olfactory Inputs to Specific Higher-Order Neurons. Neuron, 79(5), 917-931. https://doi.org/10.1016/j.neuron.2013.06.014

Liu, W. W., \& Wilson, R. I. (2013). Glutamate is an inhibitory neurotransmitter in the Drosophila olfactory system. Proceedings of the National Academy of Sciences, 110(25), 10294-10299. https://doi.org/10.1073/pnas.1220560110

MacWilliam, D., Kowalewski, J., Kumar, A., Pontrello, C., Ray, A. (2018). Signaling Mode of the Broad-Spectrum Conserved CO 2 Receptor Is One of the Important Determinants of Odor Valence in Drosophila. Neuron, 97(5), 1153-1167.e4. https://doi.org/10.1016/J.NEURON.2018.01.028

Mahr, A., \& Aberle, H. (2006). The expression pattern of the Drosophila vesicular glutamate transporter: A marker protein for motoneurons and glutamatergic centers in the brain. Gene Expression Patterns, 6(3), 299-309. https://doi.org/10.1016/J.MODGEP.2005.07.006

Marin, E. C., Jefferis, G. S. X. E., Komiyama, T., Zhu, H., \& Luo, L. (2002). Representation of the Glomerular Olfactory Map in the Drosophila Brain. Cell, 109(2), 243-255. https://doi.org/10.1016/S0092-8674(02)00700-6

Min, S., Ai, M., Shin, S. A., \& Suh, G. S. B. (2013). Dedicated olfactory neurons mediating 
623

624

625

626

627

628

629

630

631

632

633

634

635

636

637

638

639

640

641

642

643

644

645

646

647

648

649

attraction behavior to ammonia and amines in Drosophila. Proceedings of the National Academy of Sciences of the United States of America, 110(14). https://doi.org/10.1073/pnas.1215680110

Mohamed, A. A. M., Retzke, T., Das Chakraborty, S., Fabian, B., Hansson, B. S., Knaden, M., \& Sachse, S. (2019). Odor mixtures of opposing valence unveil inter-glomerular crosstalk in the Drosophila antennal lobe. Nature Communications, 10(1). https://doi.org/10.1038/s41467-019-09069-1

Münch, D., \& Galizia, C. (2016). DoOR 2.0--Comprehensive Mapping of Drosophila melanogaster Odorant Responses. Scientific Reports, 6. https://doi.org/10.1038/SREP21841

Ng, M., Roorda, R., Lima, S., Zemelman, B., Morcillo, P., Miesenböck, G. (2002). Transmission of olfactory information between three populations of neurons in the antennal lobe of the fly. Neuron, 36(3), 463-474. https://doi.org/10.1016/S0896-6273(02)00975-3

Parnas, M., Lin, A. C., Huetteroth, W., \& Miesenböck, G. (2013). Odor Discrimination in Drosophila: From Neural Population Codes to Behavior. Neuron, 79(5), 932-944. https://doi.org/10.1016/j.neuron.2013.08.006

Root, C., Semmelhack, J., Wong, A., Flores, J., Wang, J. (2007). Propagation of olfactory information in Drosophila. Proceedings of the National Academy of Sciences of the United States of America, 104(28), 11826-11831. https://doi.org/10.1073/PNAS.0704523104

Ruta, V., Datta, S. R., Vasconcelos, M. L., Freeland, J., Looger, L. L., \& Axel, R. (2010). A dimorphic pheromone circuit in Drosophila from sensory input to descending output. Nature, 468(7324), 686-690. https://doi.org/10.1038/nature09554

Schmuker, M., de Bruyne, M., Hähnel, M., \& Schneider, G. (2007). Predicting olfactory receptor neuron responses from odorant structure. Chemistry Central Journal 2007 1:1, 1(1), 1-10. https://doi.org/10.1186/1752-153X-1-11

Schmuker, M., \& Schneider, G. (2007). Processing and classification of chemical data inspired by insect olfaction. Proceedings of the National Academy of Sciences, 104(51), 2028520289. https://doi.org/10.1073/PNAS.0705683104 
650

651

652

653

654

655

656

657

658

659

660

661

662

663

664

665

666

667

668

669

670

671

672

673

674

675

676

Seki, Y., Dweck, H. K. M., Rybak, J., Wicher, D., Sachse, S., \& Hansson, B. S. (2017).

Olfactory coding from the periphery to higher brain centers in the Drosophila brain. $B M C$ Biology, 15(1), 18-22. https://doi.org/10.1186/s12915-017-0389-z

Shearin, H. K., Quinn, C. D., Mackin, R. D., Macdonald, I. S., \& Stowers, R. S. (2018). tGRASP, a targeted GRASP for assessing neuronal connectivity. Journal of Neuroscience Methods, 306, 94-102. https://doi.org/10.1016/j.jneumeth.2018.05.014

Stocker, R. F., Heimbeck, G., Gendre, N., \& De Belle, J. S. (1997). Neuroblast ablation in Drosophila P[GAL4] lines reveals origins of olfactory interneurons. Journal of Neurobiology, 32(5), 443-456. https://doi.org/10.1002/(SICI)10974695(199705)32:5<443::AID-NEU1>3.0.CO;2-5

Strutz, A., Soelter, J., Baschwitz, A., Farhan, A., Grabe, V., Rybak, J., Knaden, M., Schmuker, M., Hansson, B. S., \& Sachse, S. (2014). Decoding odor quality and intensity in the Drosophila brain. ELife, 3, e04147. https://doi.org/10.7554/eLife.04147

Tanaka, N. K., Awasaki, T., Shimada, T., \& Ito, K. (2004). Integration of chemosensory pathways in the Drosophila second-order olfactory centers. Current Biology, 14(6), 449457. https://doi.org/10.1016/j.cub.2004.03.006

Taniguchi, H., Gollan, L., Scholl, F. G., Mahadomrongkul, V., Dobler, E., Limthong, N., Peck, M., Aoki, C., \& Scheiffele, P. (2007). Silencing of neuroligin function by postsynaptic neurexins. Journal of Neuroscience. https://doi.org/10.1523/JNEUROSCI.0032-07.2007

Varela, N., Gaspar, M., Dias, S., \& Vasconcelos, M. L. (2019). Avoidance response to CO 2 in the lateral horn. PLoS Biology, 17(1), 1-26. https://doi.org/10.1371/journal.pbio.2006749

Wang, J. W., Wong, A. M., Flores, J., Vosshall, L. B., \& Axel, R. (2003). Two-photon calcium imaging reveals an odor-evoked map of activity in the fly brain. Cell, 112(2), 271-282. https://doi.org/10.1016/S0092-8674(03)00004-7

Wilson, R. I., Turner, G. C., \& Laurent, G. (2004). Transformation of Olfactory Representations in the Drosophila Antennal Lobe. Science (New York, N.Y.), 303(5656), 366-370. https://doi.org/10.1126/science.1090782 
677 Wong, A. M., Wang, J. W., \& Axel, R. (2002). Spatial representation of the glomerular map in

678 the Drosophila protocerebrum. Cell, 109(2), 229-241. https://doi.org/10.1016/S0092-

$679 \quad 8674(02) 00707-9$

680 Xing, G., Li, M., Sun, Y., Rui, M., Zhuang, Y., Lv, H., Han, J., Jia, Z., \& Xie, W. (2018).

681 Neurexin-neuroligin 1 regulates synaptic morphology and functions via the WAVE

682 regulatory complex in Drosophila neuromuscular junction. ELife, 7, 1-23.

683 https://doi.org/10.7554/eLife.30457

684 Zhang, K., Guo, J., Peng, Y., Xi, W., Guo, A. (2007). Dopamine-mushroom body circuit

685 regulates saliency-based decision-making in Drosophila. Science (New York, N.Y.),

686 316(5833), 1901-1904. https://doi.org/10.1126/SCIENCE.1137357

687

688

689

690

691

692

693

694

695

696

697 


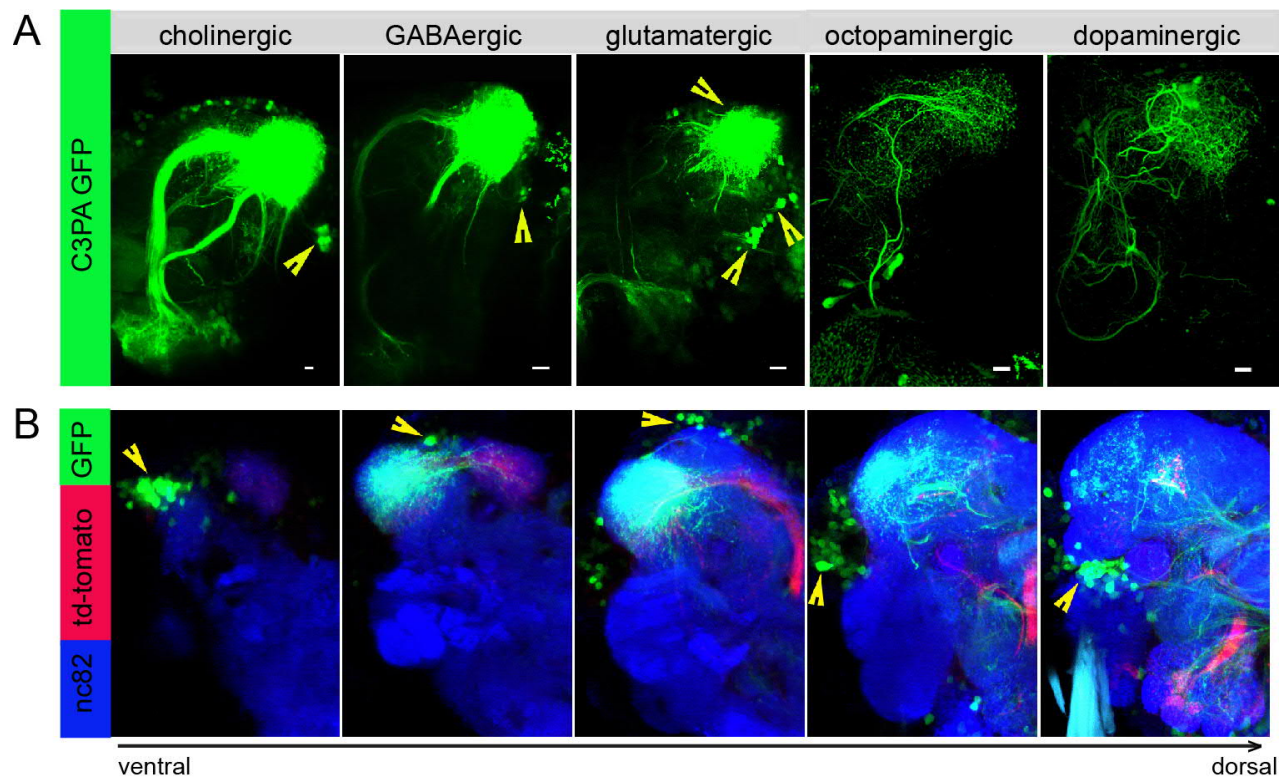

698 Figure 1: Selective labeling of LHNs based on their neurotransmitter identities. (A)

699 Photoactivation of UAS-C3PA-GFP expressed under the control of different Gal4 lines with

700 various neurotransmitter and neuromodulator identities (Cha-Gal4, GAD1-Gal4, dVGlut-Gal4, $701 \mathrm{TH}$-Gal4 and tdc2-Gal4) reveals subsets of cholinergic, GABAergic, glutamatergic, 702 octopaminergic and dopaminergic LHNs (left to right). Position of the cell bodies are demarcated 703 with yellow arrowheads. (B) Immunohistochemistry of photoactivated brains with $d V G l u t$-Gal4 704 driving UAS-C3PA-GFP reveals different cell body clusters of glutamatergic LHNs found at 705 various locations in different focal planes. GH146-QF driving QUAS-td tomato in the background 706 are depicted in red and nc82 in blue. Position of the cell bodies are shown with yellow arrowheads. $707 \quad$ Scale bars $=10 \mu \mathrm{m}$. 
bioRxiv preprint doi: https://doi.org/10.1101/2021.09.29.462361; this version posted October 1,2021. The copyright holder for this preprint (which was not certified by peer review) is the author/funder, who has granted bioRxiv a license to display the preprint in perpetuity. It is made available under aCC-BY-ND 4.0 International license.

A

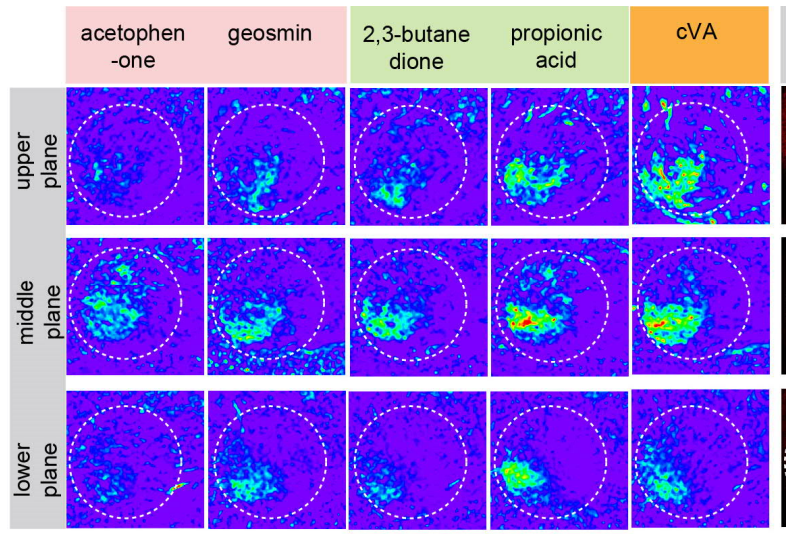

C

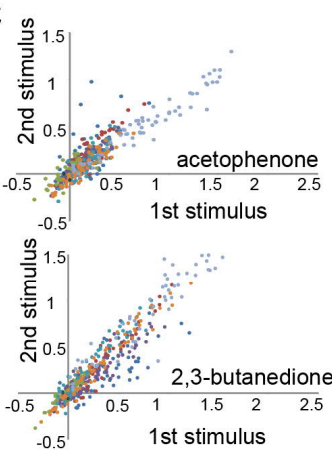

$\mathrm{F}$

음

G
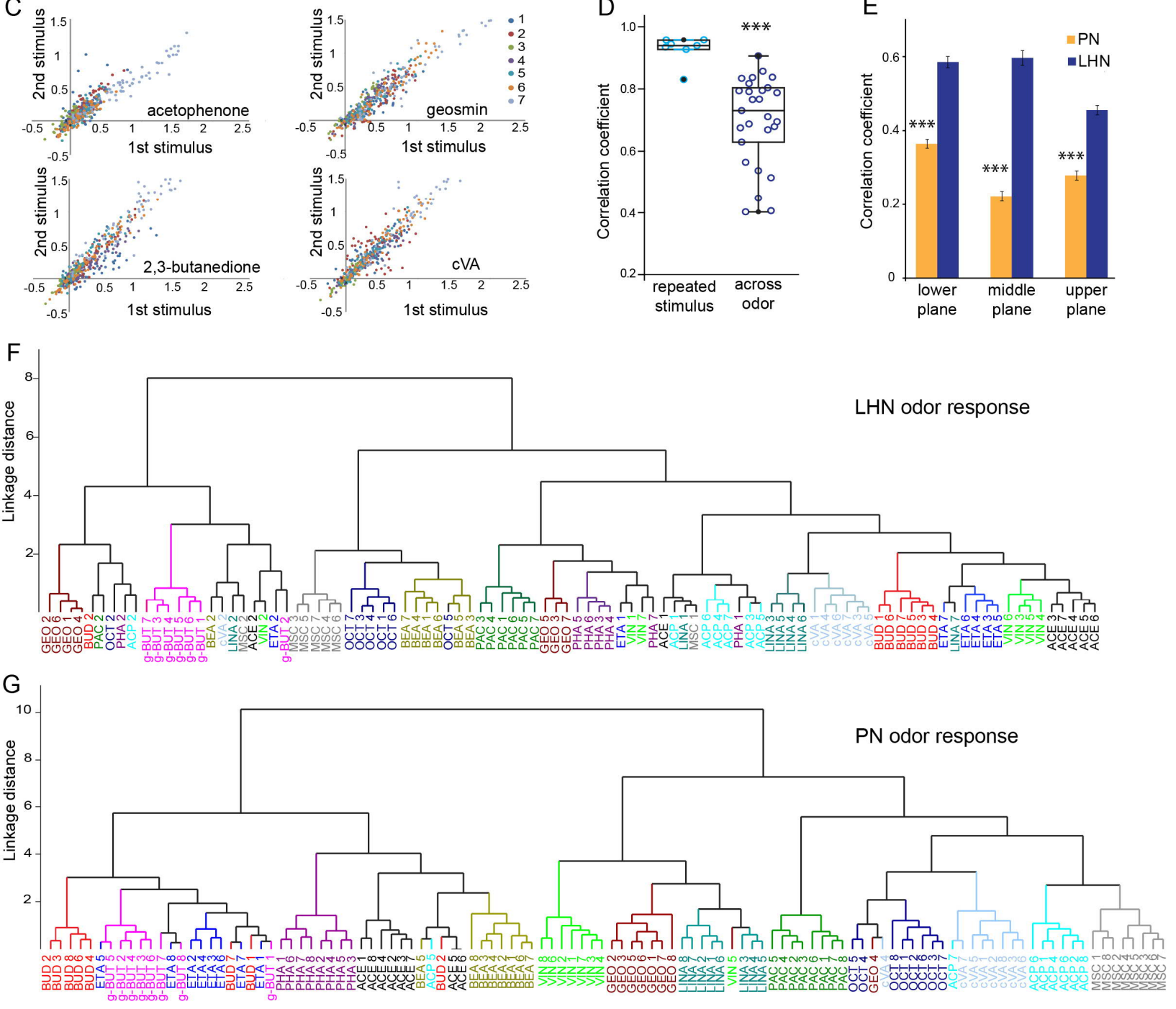

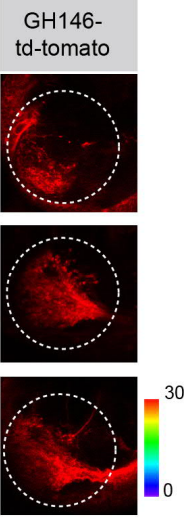

$\mathrm{B}$
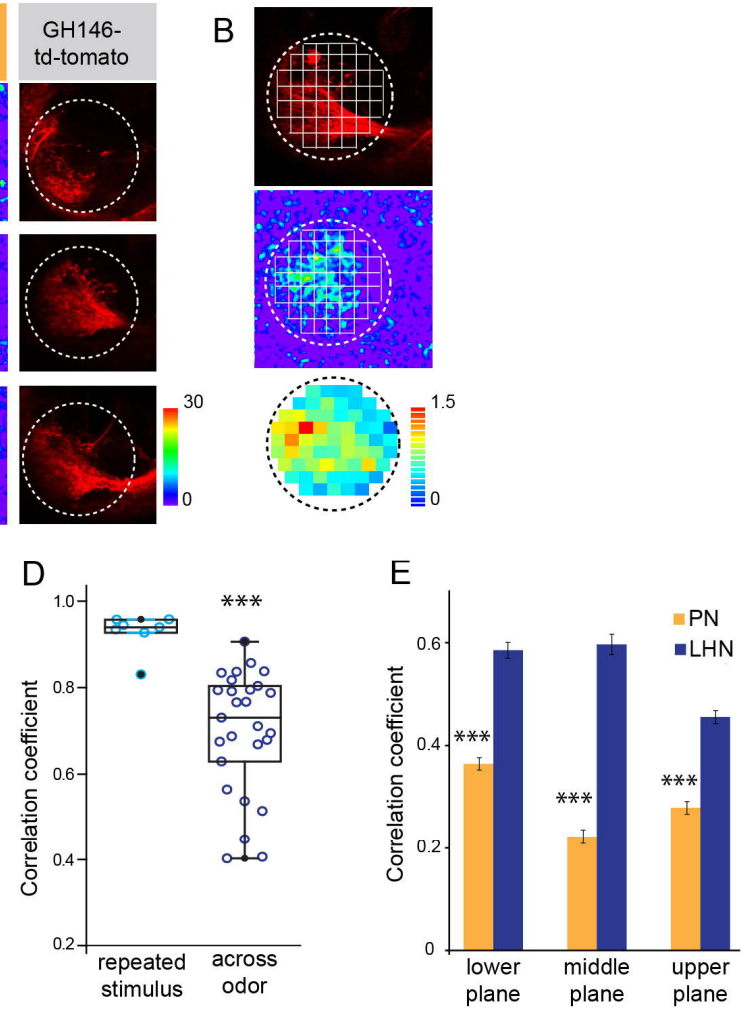

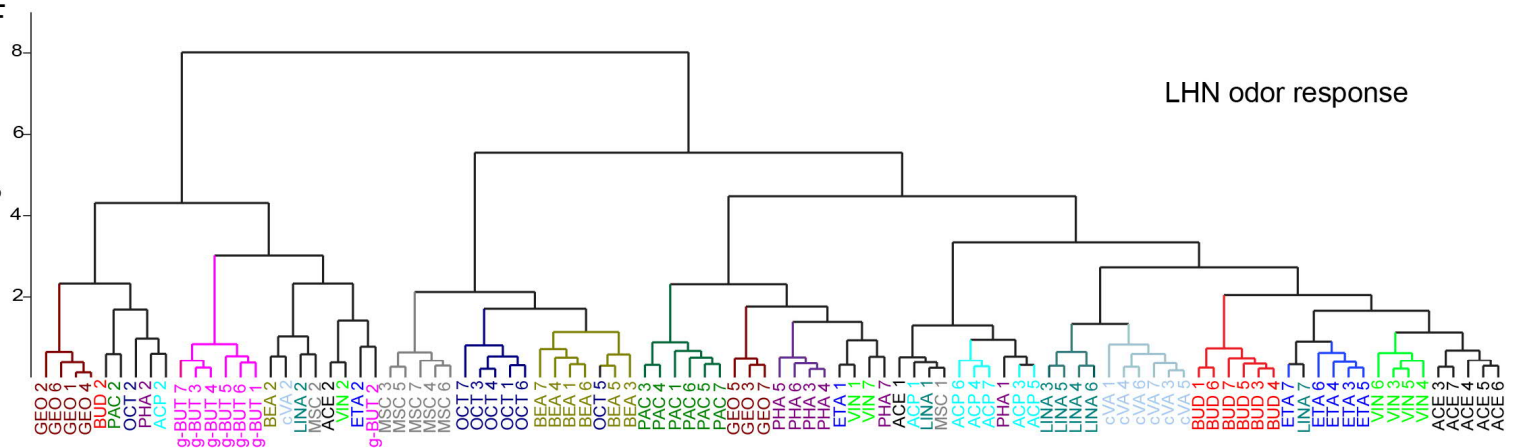

709 images of calcium responses of glutamatergic LHNs in the LH brain area in three focal planes,

710 evoked by various odors (repulsive odors: acetophenone, geosmin; attractive odors: 2,3 
711 butanedione, propionic acid; pheromone: cVA). Right panel shows the innervation pattern of uPNs

712 labeled by GH146 in three different focal planes, used as a landmark to maintain comparable focal

713 planes for functional imaging across different animals. (B) Grid approach to analyze odor-evoked

714 responses of glutamatergic LHNs. Representative images of the grid onto uPN labeling (upper

715 panel), used as a background landmark, odor-evoked responses of glutamatergic LHNs (middle

716 panel) and analyzed $\Delta \mathrm{F} / \mathrm{F}$ of each pixels from glutamatergic LHNs in the LH area (lower panel).

717 (C) Scatter plot showing calcium responses of each pixel of glutamatergic LHNs at middle plane

718 to repeated presentation of an odor stimulus. Different colored dots depict responses obtained from

719 different animals. (D) Box plot represents comparison of odor-evoked responses of glutamatergic

720 LHNs at middle plane, between repeated and across odor stimuli (student t-test, ***p < 0.001). (E)

721 Comparison of correlation coefficients of uPN and LHN responses at three different focal planes.

722 Different odors seem to be better segregated at the uPN level than at the level of LHNs (student t-

723 test, $* * * \mathrm{p}<0.001)$. (F) Cluster analysis of LHN odor responses based on linkage distance $(\mathrm{n}=7$,

724 ANOSIM, sequential Bonferroni significance, $\mathrm{p}=0.0001)$. (G) Cluster analysis of uPN odor

725 responses based on linkage distance $(n=8$; ANOSIM, sequential Bonferroni significance, $p=$

726 0.0001). Both cluster analyses show that the majority of replicates for each odor are grouped

727 together. 
A

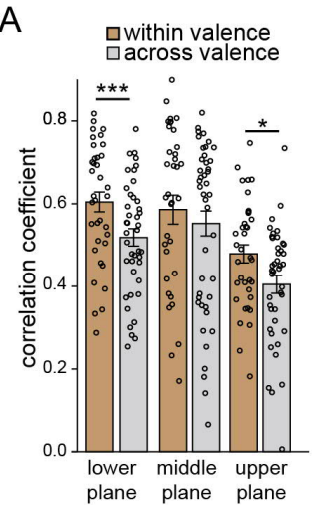

D

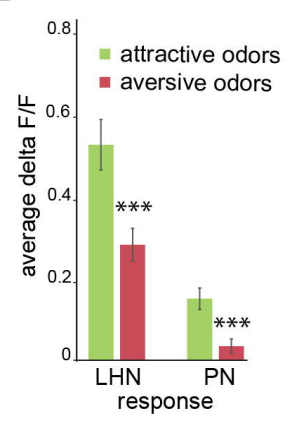

B

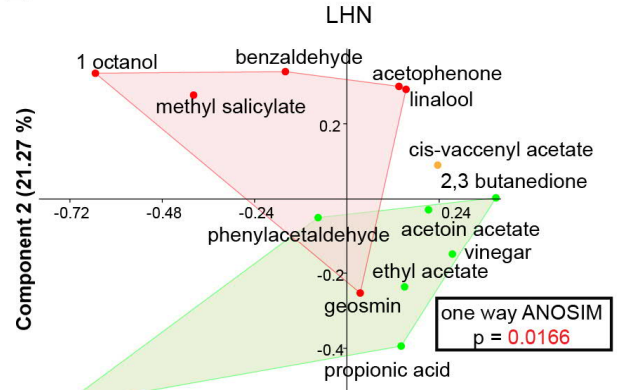

gamma- butyrolactone

Component 1 (32.405\%)

E

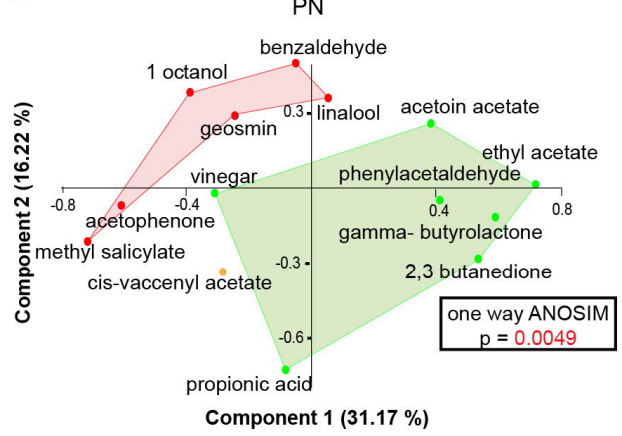

C

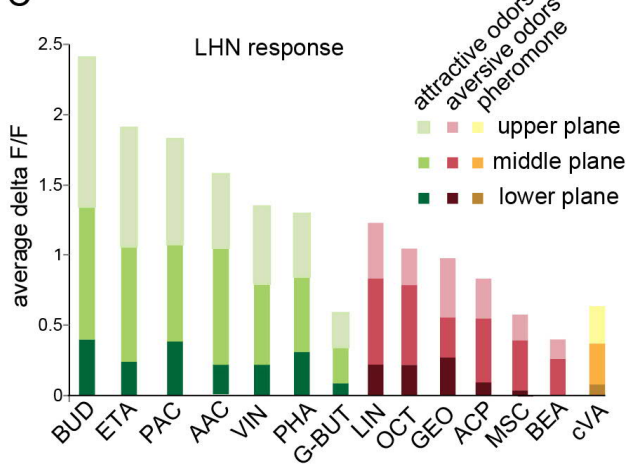

$F$

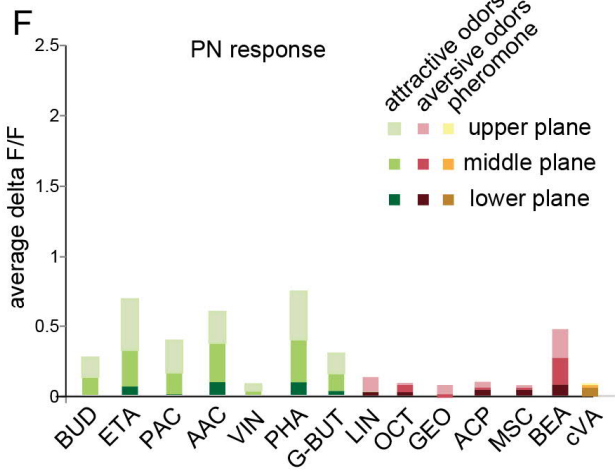

G

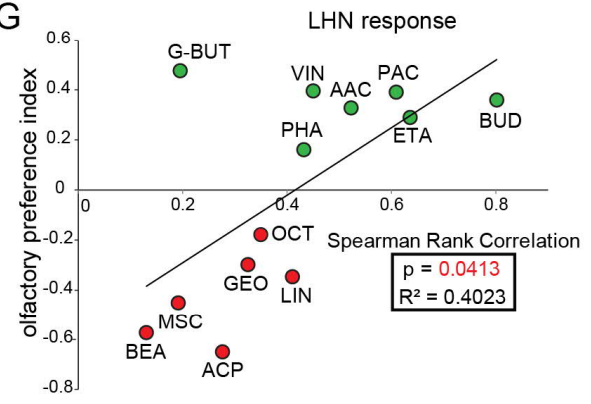

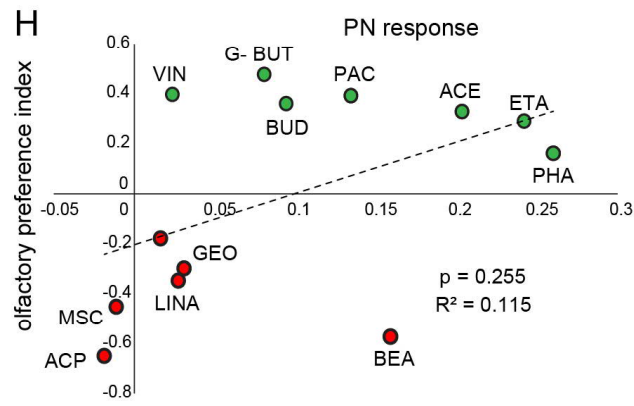
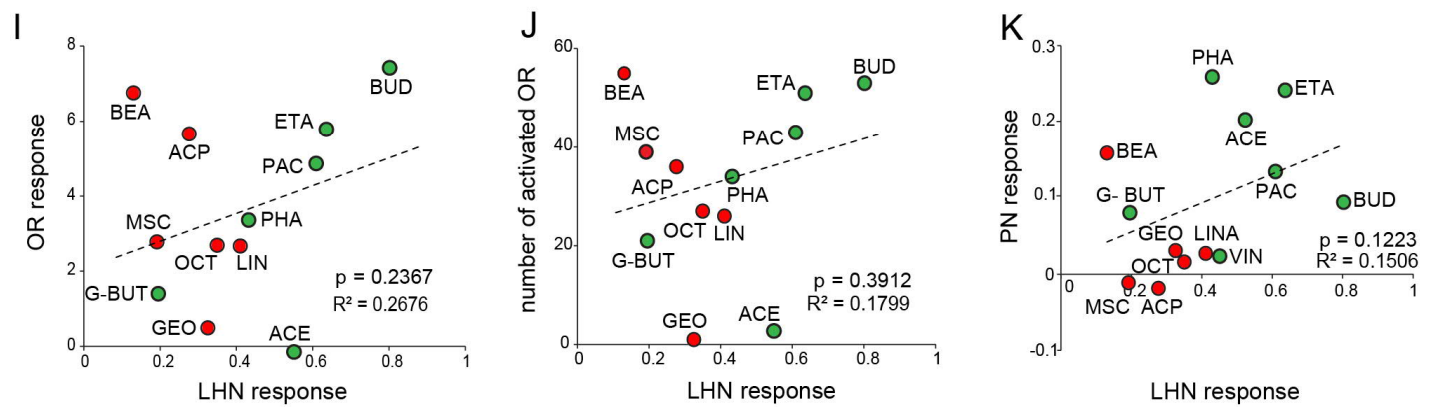

729 coefficients of odor responses of glutamatergic LHNs between the odors that share the same

730 valence (i.e. within valence) and odors having an opposing valence (i.e. across valence). Responses

731 to odors sharing the same valence are significantly more similar than those across valence (student 
732 t-test, $* * * \mathrm{p}<0.001, * \mathrm{p}<0.05)$ (B) PCA analysis of odor responses of glutamatergic LHNs shows

733 that attractive (green) and aversive (red) odors are significantly segregated in the LH (one way

734 ANOSIM, p=0.0166). (C) Response amplitudes of glutamatergic LHNs to individual attractive

735 and aversive odors. (D) Comparison of the overall response strength of glutamatergic LHNs and

736 uPNs to all attractive and aversive odors (student t-test, $* * * p<0.001)(\mathrm{E})$ PCA analysis of odor

737 responses of uPNs shows that attractive (green) and aversive (red) odors are significantly

738 segregated in the LH (one way ANOSIM, $\mathrm{p}=0.0049$ ). (F) Response amplitudes of uPNs to

739 individual attractive and aversive odors. (G) Scatter plot of olfactory preference indices of all

740 tested odors to the response strengths of glutamatergic LHNs. The solid trend line demarcates

741 significant correlation between the two parameters (Spearmann Rank Correlation, $\mathrm{p}=0.04)$. $(\mathrm{H})$

742 Scatter plot of olfactory preference indices of all tested odors to the response strengths of uPNs,

743 which does not show a significant correlation (dotted trend line). (I) Scatter plot of the response

744 strength of glutamatergic LHNs for individual odors to the overall OSN activity. (J) Scatter plot

745 of the response strength of glutamatergic LHNs for individual odors to the number of ORs

746 activated. (K) Scatter plot of the response strength of glutamatergic LHNs and the response

747 strengths of uPNs for individual odors. 


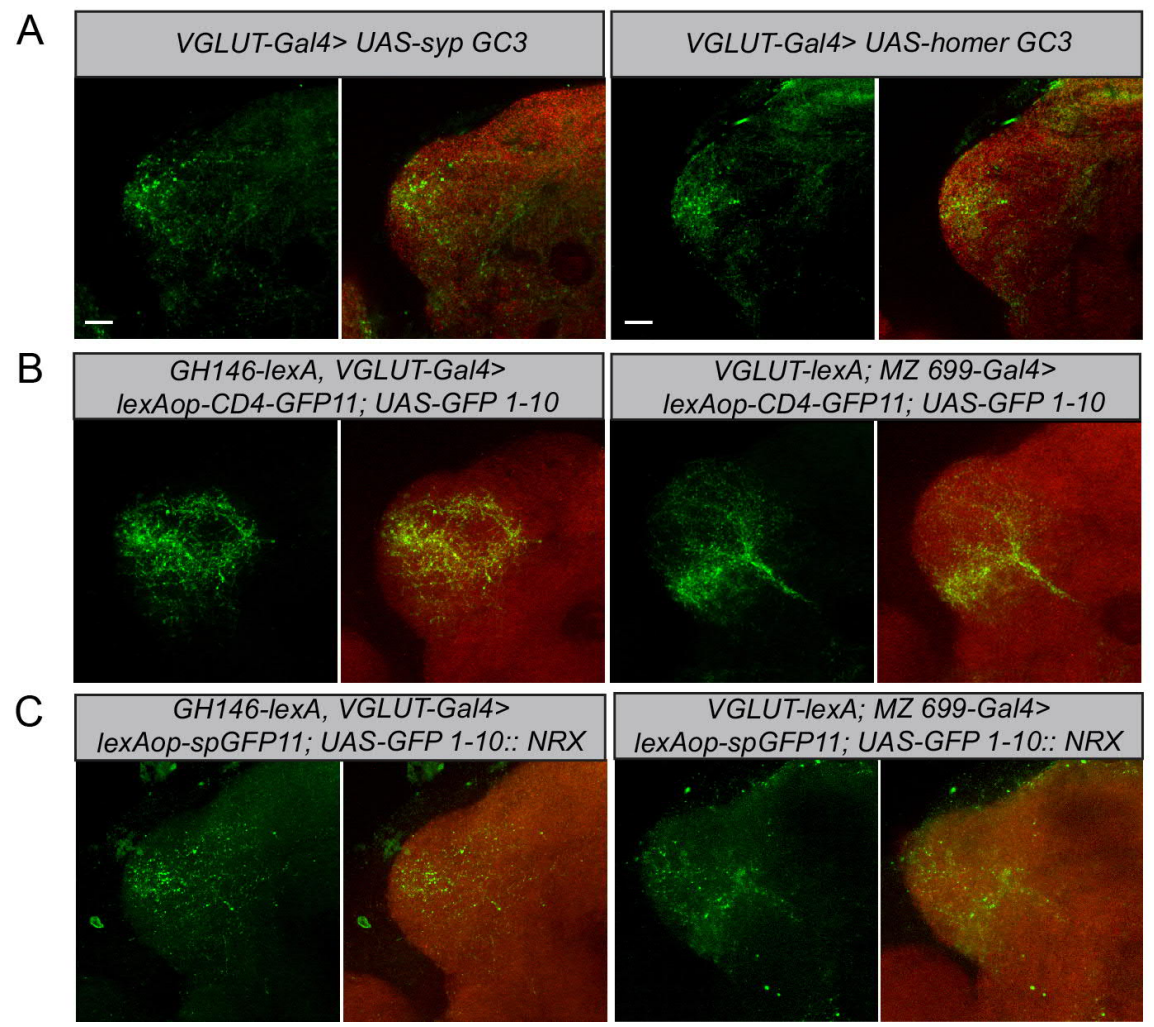

749 mPNs. (A) Expression of syp GC3 (left panel) and homer GC3 (right panel) in glutamatergic

750 LHNs suggesting the presence of pre- as well as postsynapses in the LH. (B) Membrane-targeted

751 CD4-GRASP between glutamatergic LHNs and uPNs (left panel) or mPNs (right panel). (C) The 752 synaptic protein neurexin tagged to GRASP (Nrx-GRASP) was employed to confirm synaptic 753 connectivity of glutamatergic LHNs with uPNs (left panel) or mPNs (right panel) in the LH. Scale $754 \quad$ bars $=10 \mu \mathrm{m}$. 
bioRxiv preprint doi: https://doi.org/10.1101/2021.09.29.462361; this version posted October 1, 2021. The copyright holder for this preprint (which was not certified by peer review) is the author/funder, who has granted bioRxiv a license to display the preprint in perpetuity. It is made available under aCC-BY-ND 4.0 International license.

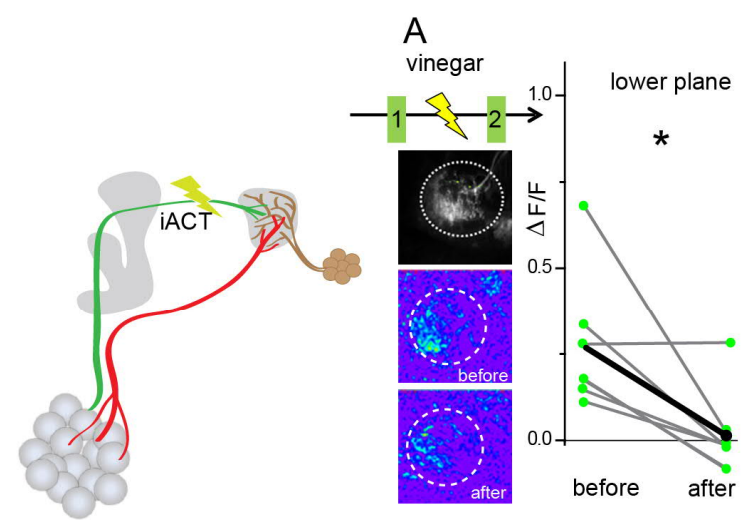

B

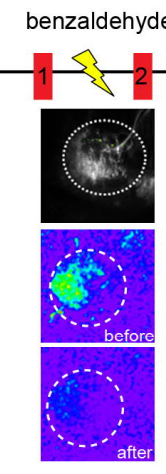

C

vinegar
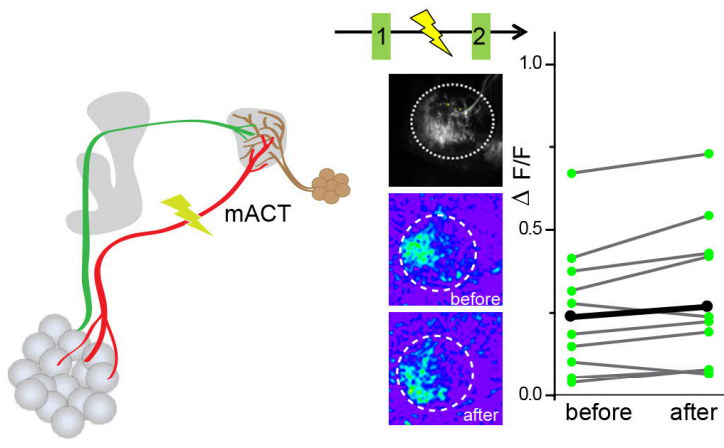

$\mathrm{D}$

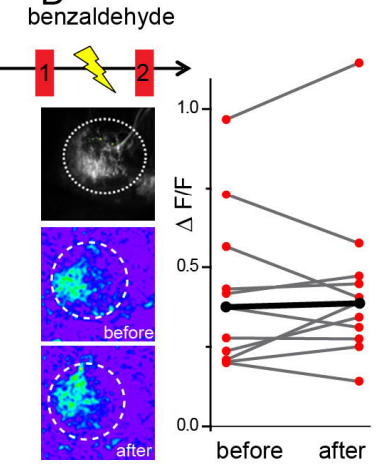

$A^{\prime}$

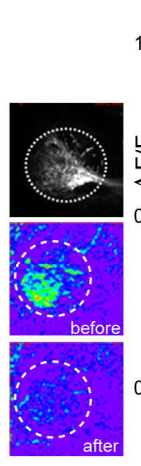

B'

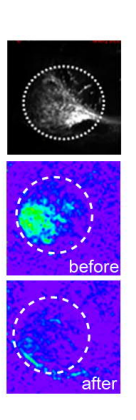

$C^{\prime}$

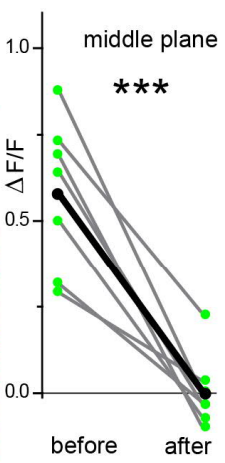

A"
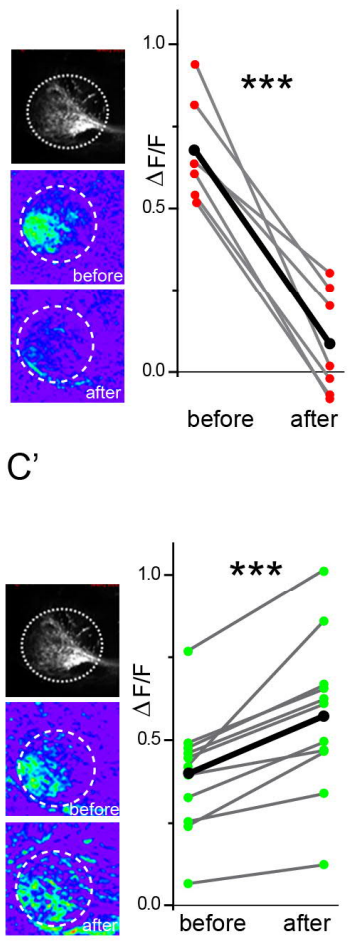

D'

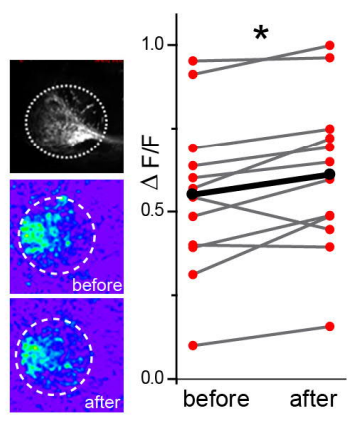

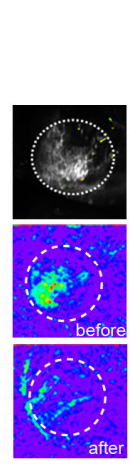

B"
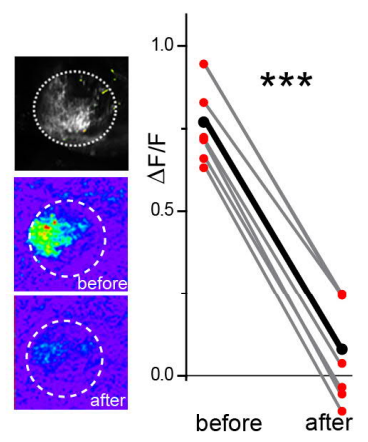

C"
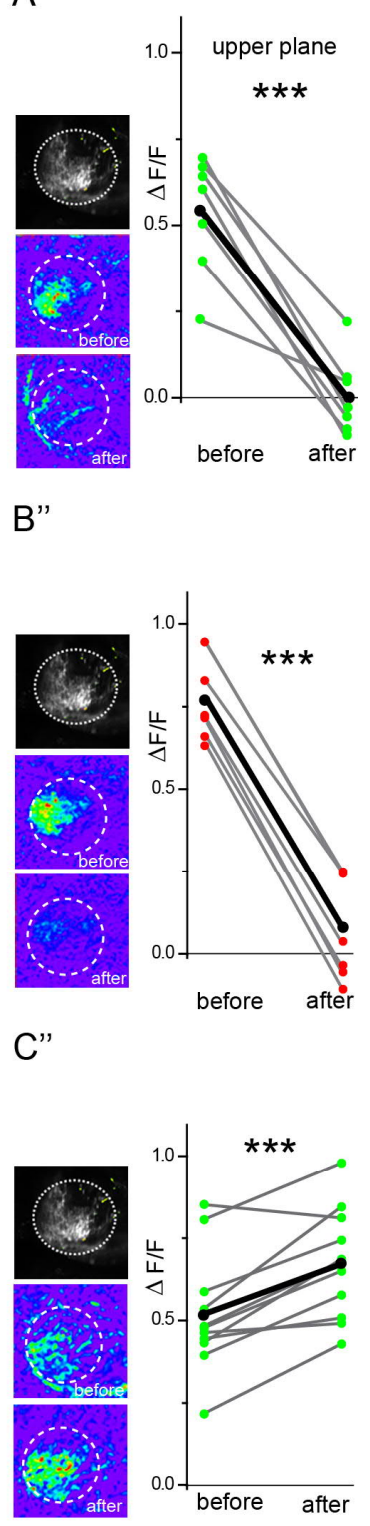

D"
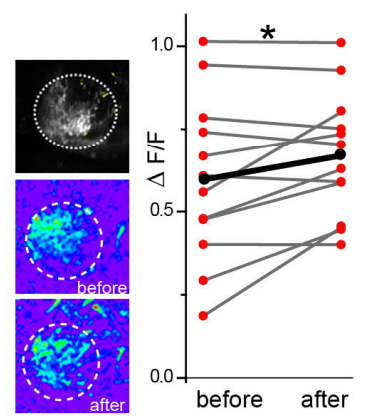
755 Figure 5: Glutamatergic LHNs receive major excitatory input from uPNs and an odorant

756 selective inhibition from mPNs. Upper panel schematic represents the experimental approach:

757 the iACT tract was laser transected while odor-evoked responses were monitored from

758 glutamatergic LHNs. (A, A' and A') Representative images and graphical comparison of

759 response evoked by vinegar in glutamatergic LHNs before and after laser transection of the

760 iACT, across three different planes. (B, B' and B')' Representative images and graphical

761 comparison of response evoked by benzaldehyde in glutamatergic LHNs before and after laser

762 transection of the iACT. Lowe panel schematic represents the experimental approach: the mACT

763 tract was laser transected while odor-evoked responses were measured from glutamatergic

764 LHNs. (C, C' and C', ) Representative images and graphical comparison of vinegar-evoked

765 responses of glutamatergic LHNs before and after laser transection of the mACT. (D, D' and

766 D'). Representative images and graphical comparison of benzaldehyde-evoked response of

767 glutamatergic LHNs before and after laser transection of the mACT (paired t-test $* * * p<0.001$,

$768 * \mathrm{p}<0.05)$. 

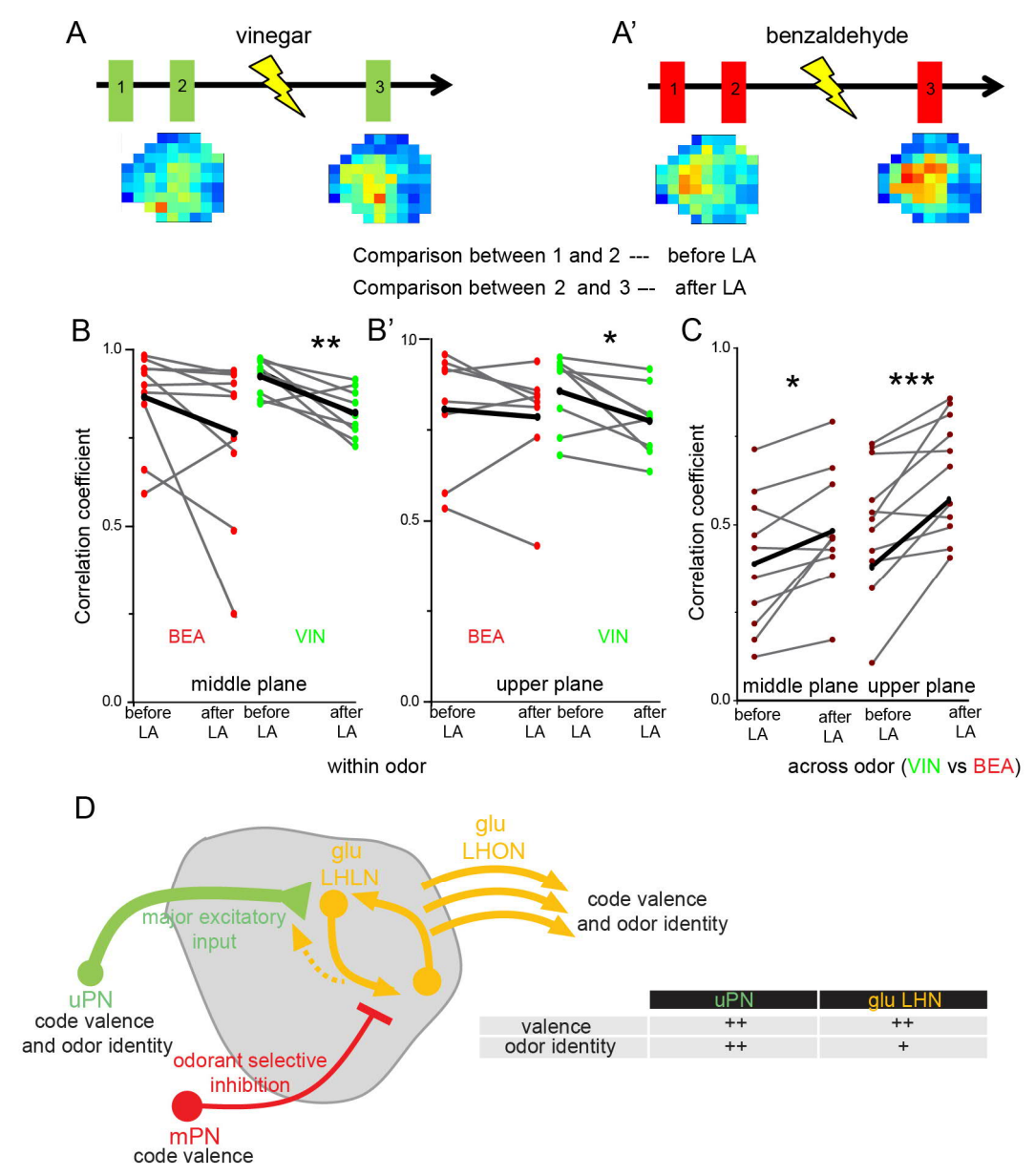

Figure 6: mPN-mediated inhibition facilitates odor specificity in glutamatergic LHNs. (A and

770 A') Schematic represents the experimental approach: odor-evoked responses of glutamatergic

771 LHNs were monitored to repeated odor presentations (1 and 2) before and after laser transection

772 of the mACT (3) for the odors vinegar and benzaldehyde. (B and B') Comparison of the correlation

773 coefficients between the repeated odor responses before laser ablation to those after the transection

774 for vinegar and benzaldehyde (within odor comparison). (C) Comparison of the correlation

775 coefficients across vinegar and benzaldehyde before and after microlesion (across odor

776 comparison; paired t-test, ${ }^{* * *} \mathrm{p}<0.001,{ }^{*} \mathrm{p}<0.005,{ }^{*} \mathrm{p}<0.05$ ). (D) Schematic summarizing the

777 observed connectivity between PNs and glutamatergic LHNs in the LH: Glutamatergic LHNs

778 consists of both, LHLNs and LHONs. uPNs (green) provide the major excitatory input to

779 glutamatergic LHNs (yellow) while they might also receive feedback input from LHNs. mPNs

780 provide an odorant selective inhibition to glutamatergic LHNs. mPNs are known to encode odor

781 valence in the LH (Strutz et al., 2014), uPNs and glutamatergic LHNs encode odor identity as well 
782 as odor valence. The table illustrates that uPNs encode an improved odor identity than postsynaptic

783 glutamatergic LHNs whereas hedonic valence is equally maintained at the level of uPNs and 784 glutamatergic LHNs in the LH.
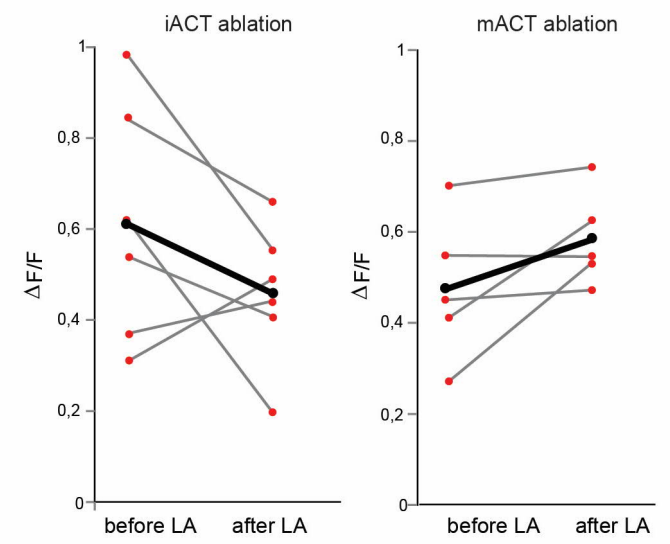

785 Figure 5- figure supplement 1: Laser transaction does not affect activity of glutamatergic

786 LHNs in the intact brain hemisphere. Left panel shows the comparison of benzaldehyde-evoked 787 activity in glutamatergic LHNs in the intact hemisphere, before and after laser ablation of the iACT 788 in the other, treated brain hemisphere (paired t-test, $\mathrm{p}=0.19$ ). Right panel shows the comparison 789 of benzaldehyde-evoked activities in glutamatergic LHNs in the intact hemisphere, before and 790 after laser ablation of the mACT in the other hemisphere (paired t-test, $p=0.25$ ). 